\title{
Self-Care Skills
}

National Cancer Institute

\section{Source}

National Cancer Institute. Self-Care Skills. NCI Thesaurus. Code C121196.

An assessment of the Adaptive Behavior Assessment System where self-care skills such as eating, dressing, bathing, toileting, grooming, and hygiene are evaluated. 